\title{
Grado de desarrollo de los portales periodísticos españoles de radio y televisión
}

\section{Rosana López Carreño}

Grupo de Tecnologías de la Información, Universidad de Murcia (España)

\subsection{Resumen}

Se analizan los elementos que componen los portales periodísticos de radio y televisión españoles, identificando su estructura y comparando su oferta de productos y servicios, según el medio de comunicación. Se comparan los resultados con el modelo de portal periodístico de prensa desarrollado por el grupo de investigación del autor, de manera que se puedan esbozar las tendencias actuales de productos y servicios presentes en el periodismo en línea español, construyendo un modelo general de referencia para cualquier tipo de portal periodístico.

Palabras clave: Portales periodísticos. Servicios de valor añadido. Periodismo electrónico. Radio. Televisión.

\subsection{Abstract}

The elements that conform Spanish radio and television portals. Their structure is analyzed and the similarities and differences in their supply of products and services are compared. The results are compared with the model of journalistic portal developed by the authors' research group, so that new trends in products and services in the Spanish online media can be outlined and a new reference model for any form of media portal can be developed.

Keywords: Mass media portals. Value added services. Radio. Television. Digital journalism.

\section{Introducción}

Tanto en el ámbito nacional como en el internacional, venimos asistiendo en la actualidad a la consolidación de los portales periodísticos en Internet. En los últimos meses, los principales grupos de comunicación de nuestro país están realizando profundas transformaciones en la estructura y en el contenido de sus distintos sitios web, incorporando una mayor cantidad de productos y servicios y confiriéndoles una mayor interactividad con los usuarios. Si bien el gran público lo que percibe es la puesta en marcha de los servicios de pago en estos portales Scire. $10: 2$ (jul.-dic. 2004) 115-122. 


\section{Rosana López Carreño}

- hasta hace poco enteramente gratuitos - , en realidad no resulta menos importante la evolución de sus componentes básicos: productos informativos (PI), productos documentales (PD) y servicios de valor añadido (SVA).

Un portal periodístico es un sitio web dónde se concentran información, productos y servicios, y que pertenece a un medio de comunicación social, sea de prensa, radio o televisión. No abundan los estudios detallados sobre la estructura de estos portales, sino más bien una serie de presentaciones generales de los mismos y de su grado de desarrollo. La mayoría de los autores coinciden en afirmar que los portales periodísticos de prensa se encuentran en un nivel más avanzado.

El actual desarrollo de los portales de prensa ha sido el objeto del estudio de nuestra tesis doctoral (López Carreño, 2003) y de algunos trabajos previos o paralelos (López Carreño y Rodríguez Muñoz, 2003; López Carreño y Martínez Méndez, 2002; Martínez Rodríguez, 2003). Los resultados de estos trabajos permiten afirmar la supremacía de los PI sobre los PD - resultado lógico, si se tiene en cuenta el alto grado de vinculación del producto informativo y la práctica periodística-, así como que el nivel de los SVA es heterogéneo y altamente dependiente de la importancia del grupo de comunicación al cual está ligado el portal periodístico. El estar ligado a grandes grupos de comunicación es uno de los factores determinantes, ya que los portales asociados a los pequeños grupos de comunicación se encuentran escasamente desarrollados, siendo pocos los que llegan a un nivel de portal básico.

El objetivo del presente trabajo es trasladar estos procesos de análisis al campo de los portales periodísticos de radio y TV, con el objeto de conocer su estado de desarrollo y verificar si existe un patrón evolutivo común, o si, por el contrario, este proceso de implantación es variado y disperso. Sólo entonces se podrá presentar una panorámica general del estado de la cuestión del periodismo electrónico español, basado en un método descriptivo que permita determinar sus fortalezas y debilidades. Paralelamente, el nuevo contexto dónde se va a desarrollar este estudio permitirá averiguar si las herramientas de análisis que hemos desarrollado previamente para los portales de origen prensa, básicamente la Guía de observación y el Modelo de referencia de portal periodístico (López Carreño, 2002), siguen siendo útiles para todas las modalidades de portal periodístico.

\section{Portales periodísticos: modalidades y componentes}

Dentro de la vorágine existente en torno a los conceptos y terminologías de la web, y más concretamente alrededor de los portales, resulta preciso señalar el desarrollo de un nuevo modelo de portal: el portal periodístico. El surgimiento de estos portales, vinculados con los medios de comunicación social tradicionales 
o con aquéllos desarrollados específicamente para la web, es debido, fundamentalmente a la consolidación de los portales como los principales puntos de acceso a la web para los usuarios, y a la necesidad de inserción de los medios de comunicación tradicionales en el nuevo panorama comunicacional, impulsado por Internet.

Los portales periodísticos, dependiendo del punto de vista, se pueden clasificar: a) según la información que tratan: - generalistas o temáticos; b) según el ámbito geográfico que cubren - internacionales, nacionales, regionales y locales-; c) según el diseño web del portal - modelo genérico o específico; y d) según su origen o medio de comunicación del que proceden — prensa, radio, televisión o Internet-. Como se ha señalado, en la estructura de estos portales se identifican tres grupos de componentes básicos: los productos informativos (PI), los productos documentales (PD) y los servicios de valor añadido (SVA), siendo el eje principal el contenido, que es canalizado a través de estos componentes y en diversas modalidades.

\section{Metodología.}

El presente análisis se realiza sobre un conjunto de portales periodísticos de radio y TV de ámbito nacional en idioma español (tabla I), a los que se ha aplicado individualmente la citada guía de observación desarrollada para el estudio

\begin{tabular}{|l|l|l|}
\hline \multirow{4}{*}{ Canal } & \multicolumn{2}{|c|}{ Uenominación } \\
\hline \multirow{5}{*}{ TV } & Cadena 100 & http://www.cadena100.es \\
\cline { 2 - 3 } & Cope & http://www.cope.es \\
\cline { 2 - 3 } & Los 40 principales & http://www.cadena40.es \\
\cline { 2 - 3 } & Onda Cero & http://www.ondacero.es \\
\cline { 2 - 3 } & Radio Nacional de España & http://www.rtve.es/rned \\
\cline { 2 - 3 } & & ndex.htm \\
\cline { 2 - 3 } & Radiocable & http://www.radiocable.com \\
\cline { 2 - 3 } & Ser & http://www.cadenaser.es \\
\hline \multirow{5}{*}{ Radio } & TV & http://www.rtve.es \\
\cline { 2 - 3 } & Antena 3 & http://www.antena3tv.com \\
\cline { 2 - 3 } & Tele 5 & http://www.telecinco.es \\
\cline { 2 - 3 } & Canal + & \\
\cline { 2 - 3 } & CNN ESPAÑOL & \\
\cline { 2 - 3 } & &
\end{tabular}

Tabla I. Conjunto de portales estudiados

Scire. $10: 2$ (jul.-dic. 2004) 115-122. 
de los portales de prensa. Esta guía, convenientemente adaptada, ha permitido delimitar, identificar y clasificar los principales elementos que los componen, con el fin de llevar a cabo su posterior comparación y eventual ponderación del nivel de desarrollo en el que se encuentran. En concreto se han analizado siete portales periodísticos de radio y cinco de TV. El estudio fue realizado entre los meses de septiembre y octubre de 2003.

\section{Características de los portales de radio}

Tras el análisis de estos portales (figura 1), se observa que el volumen de productos en esta modalidad de portal periodístico es menor que en los de prensa, siendo la media seis productos, de los cuales cuatro son productos informativos y dos productos documentales. Entre los productos informativos presentes en los portales de radio examinados, aparte de las "Noticias", destacan en orden de frecuencia: "Especiales", "Programación", "Entrevistas" y "Tiempo". Con cinco productos informativos, los portales periodísticos "COPE" y "SER" sobresalen en su oferta, frente al resto de portales estudiados. En relación con los productos documentales presentes en los portales de radio, predominan las "Guías de recursos" y los "Resúmenes o notas de prensa". En cuanto al producto documental "Críticas/Reseñas" se trata de un producto característico de los de portales periodísticos de radio que no se oferta en el resto. El portal mantenido por Los 40 Principales es el que mayor número de productos documentales oferta, esto es, cuatro.

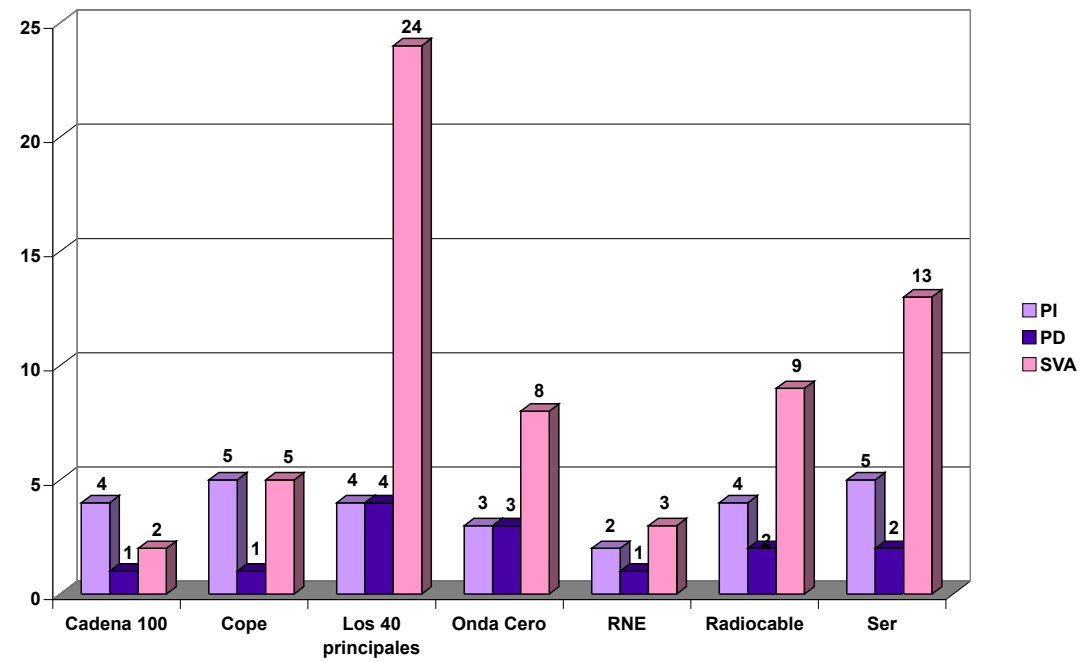

Figura 1. Distribución de elementos componentes en los portales de origen radio

Scire. $10: 2$ (jul.-dic. 2004) 115-122. 
Los servicios de valor añadido se han clasificado en ocho categorías (López Carreño, 2003), debido a su elevado número y heterogeneidad. Tras analizar los portales periodísticos de origen radio, destacan por su frecuencia de aparición los siguientes: acceso a la información — "Portales verticales", "Buscador de noticias" y "Mapa web" - ; interactivos — "Foros" - ; de participación "Sugerencias" y "Encuestas"-; multimedia — "Radio digital"; personalizados _-"WAPnews"-; de Entretenimiento - "Horóscopos"; comerciales _-"Viajes online"-; siendo nula la presencia de SVA complementarios. De entre los portales analizados destaca sobremanera el portal Los 40 Principales, seguido muy de lejos por el portal SER.

\section{Características de los portales periodísticos de TV}

Del mismo modo que ocurre con los portales de radio, se observa que la presencia de productos en esta modalidad de portal periodístico es menor que en los de prensa, siendo también inferior que en el caso de la radio, fijándose la media en cuatro productos, de los cuales tres son productos informativos y únicamente uno es documental. De entre los productos informativos presentes en los portales de TV examinados, aparte de las "Noticias", destacan en orden de frecuencia: "Programación", "Tiempo" y "Especiales". Con una frecuencia de aparición de cuatro productos informativos, los portales Canal+ y CNN Español sobresalen en sus ofertas de este tipo de productos frente al resto de portales analizados. En cuanto a la presencia de productos documentales en los portales de TV, el produc-

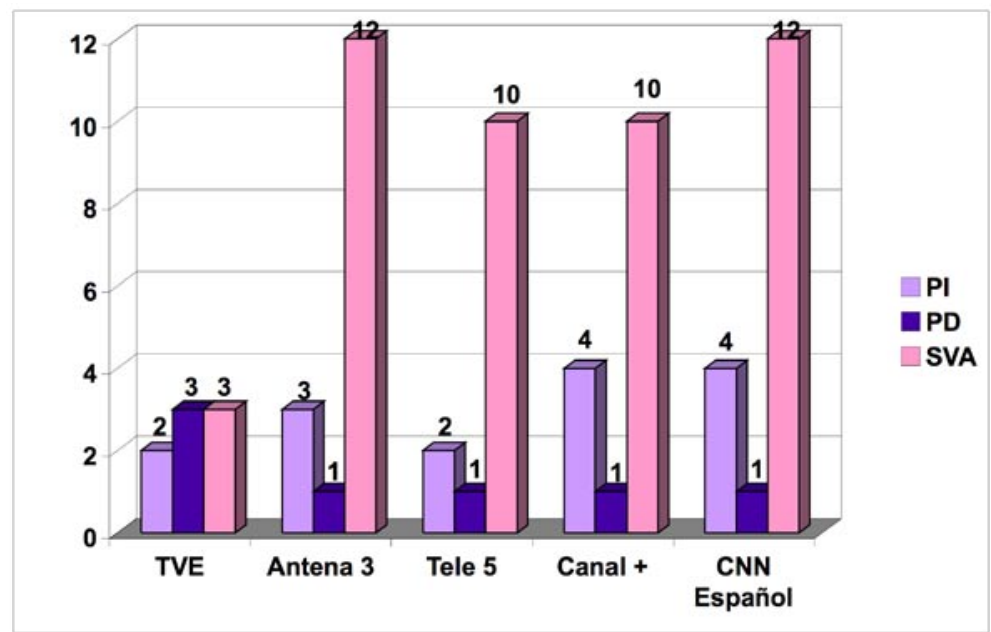

Figura 2. Distribución de elementos componentes en los portales de origen TV

Scire. $10: 2$ (jul.-dic. 2004) 115-122. 
to "Resúmenes o notas de prensa" es el único ofertado en todos los portales de TV. En este caso, el portal TVE, con un número de tres productos documentales, es el que más destaca en su oferta.

Del mismo modo que en el caso de portales de radio, los servicios de valor añadido (SVA) fueron agrupados en las ocho categorías establecidas, destacando por su frecuencia de aparición los siguientes: acceso a la información - "Portales verticales", "Buscador de noticias" y "Mapa web"-; interactivos — "Chat"-; de participación - "Sugerencias" y "Encuestas" - ; multimedia - "Radio digital"; personalizados - "MailNews"-; de entretenimiento - "Horóscopos"; comerciales - "Tienda/s"; siendo nula la presencia de SVA complementarios. Como se puede apreciar, los portales de TV que destacan en su oferta general de SVA son Antena 3 y CNN Español, seguidos muy de cerca por Tele 5 y Canal+.

\section{Análisis comparativo de portales periodísticos}

Tomando como base el modelo de referencia de portal periodístico establecido a partir del estudio de portales periodísticos de prensa (López Carreño, 2003), y a partir de los resultados obtenidos en este trabajo, es posible establecer tanto el perfil de portal periodístico de radio, como del perfil de portal periodístico de $T V$ de nuestro país.

\begin{tabular}{|c|c|c|}
\hline & TV & Radio \\
\hline $\bar{\alpha}$ & $\begin{array}{l}\text { Programación } \\
\text { Tiempo } \\
\text { Especiales }\end{array}$ & $\begin{array}{l}\text { Especiales } \\
\text { Programación } \\
\text { Entrevistas } \\
\text { Tiempo }\end{array}$ \\
\hline$\stackrel{\circ}{a}$ & Resúmenes o notas de prensa & $\begin{array}{l}\text { Guías de recursos } \\
\text { Resúmenes o notas de prensa }\end{array}$ \\
\hline$\stackrel{\nwarrow}{\lessgtr}$ & $\begin{array}{l}\text { Portales verticales. Buscador de } \\
\text { noticias. Mapa web } \\
\text { Chat } \\
\text { Sugerencias, Encuestas } \\
\text { Radio digital } \\
\text { MailNews } \\
\text { Horóscopos } \\
\text { Tienda/s }\end{array}$ & $\begin{array}{l}\text { Portales verticales. Buscador de } \\
\text { noticias. Mapa web } \\
\text { Foros } \\
\text { Sugerencias. Encuestas } \\
\text { Radio digital } \\
\text { WAPnews } \\
\text { Horóscopos } \\
\text { Viajes online }\end{array}$ \\
\hline
\end{tabular}

Figura 3. Perfiles de portales periodísticos prensa y TV

Estas dos modalidades de portal se encuentran en un nivel básico de desarrollo con respecto a la modalidad de portal de prensa. Además, puede observarse fácilmente que los perfiles de dichas modalidades de portal periodístico (radio 
y TV) son prácticamente idénticos. Los portales de radio han implementado un producto informativo y un producto documental más que los de televisión - "Entrevistas" y "Guías de Recursos"-, y las diferencias entre los servicios de valor añadido son mínimas, prácticamente de matiz - "Foros", "Wapnews" y "Viajes" en radio, frente a "Chat", "Mailnews" y "Tiendas" en televisión-.

\section{Conclusiones}

El resultado del párrafo anterior sirve de base para el enunciado de una de las principales conclusiones de nuestro trabajo, que corrobora la hipótesis del mayor desarrollo de los portales de prensa frente a los de radio y TV, que además presentan una gran analogía en su implantación. Este incipiente nivel de desarrollo no es óbice para prever que en breve plazo estos portales seguirán el camino marcado por los portales de origen prensa, y los cambios en este tipo de portal se vienen produciendo de forma paulatina y progresiva. De hecho, el portal que más oferta de SVA presenta, Los 40 principales, se encuentra ya en un nivel intermedio de desarrollo. Al igual que sucede en los portales periodísticos de prensa, predominan los productos informativos frente a los documentales, lo que confirma que aún queda por recorrer un largo trecho en lo que se refiere a la presencia de este tipo de productos en los portales periodísticos. La presencia de 32 servicios de valor añadido en los portales de radio y de 26 en los de TV, frente a los 44 que se identificaron en los portales de prensa, muestra el grado superior de desarrollo de éstos últimos en este campo. En los portales periodísticos de radio y TV, destacan por su frecuencia de uso las categorías de servicios de valor añadido de acceso a la información y de participación. De modo semejante sucede en los portales de origen prensa donde los SVA de acceso a la información se alzan como los servicios más ofertados, aunque los interactivos se colocan por delante de los de participación.

El análisis de los componentes de estos portales nos permite afirmar que el modelo empírico de portal periodístico establecido en nuestra tesis doctoral es plenamente válido para los portales de origen radio o TV, constituyéndose, pues, en un valioso instrumento de medida para conocer el nivel alcanzado por un portal periodístico, verificándose la existencia de un patrón común para la implantación de los portales periodísticos con independencia de su origen.

Otro de los aspectos a destacar es el predominio del formato textual frente al multimedia en los portales periodísticos, a pesar de ser medios de comunicación cuyo vehículo de difusión, Internet, posee un potencial multimediático de explotación insospechada en el ámbito comunicacional. Esta carencia cobra especial importancia cuando gran parte de la información que se maneja en las emisoras de televisión posee formato multimedia, que no parecen saber reutilizar posteriormente en sus portales. Sin duda alguna, las limitaciones actuales de ancho de

Scire. $10: 2$ (jul.-dic. 2004) 115-122. 
banda en las líneas de comunicaciones constituyen un factor decisivo en la poca utilización del vídeo (streaming o diferido) en estos portales.

Como conclusión final, parece observarse que el desarrollo de los portales periodísticos sigue un patrón común, no detectándose divergencias relacionadas con el origen del mismo, que pueden deberse principalmente a la gran aceptación que el modelo de referencia de portal periodístico de prensa tiene tanto entre los usuarios como entre los profesionales.

\section{Referencias.}

López Carreño, R. (2003). Análisis de los portales periodísticos españoles. Taxonomía de sus elementos componentes. Alicante: Biblioteca Virtual Miguel de Cervantes, 2003. $<$ http://www.cervantesvirtual.com/FichaObra.html?Ref=10572>. Consulta: $22 \mathrm{de}$ diciembre de 2003. Tesis doctoral.

López Carreño, R. ; Martínez Méndez, F. J. (2002). La importancia de los productos documentales dentro de la gestión de los e-contents en los portales periodísticos. [En CD-ROM]. // CALSI Workshop. Valencia, 2002. [En línea] E-Lis, Italia. <http: //eprints.rclis.org/archive/00000525/>. Consulta: 23 de diciembre de 2003.

López Carreño, R. y Rodríguez Muñoz, J. V. (2003a). Los servicios de valor añadido en los portales periodísticos: Una propuesta de clasificación. // Scire: Representación y Organización del Conocimiento. 9:2 (2003) 111-122.

Martínez Rodríguez, Lara (2003). Análisis de los portales periodísticos de ámbito regional y local. Murcia: Facultad de Comunicación y Documentación, Universidad, septiembre 2003. Proyecto de fin de carrera. 